\title{
Comparative Operando XPS and SEM Spatiotemporal Potential Mapping of Ionic Liquid Polarization in a Coplanar Electrochemical Device
}

\author{
Sefik Suzer,* Evgheni Strelcov, and Andrei Kolmakov*
}

Cite This: Anal. Chem. 2021, 93, 13268-13273

Read Online

ACCESS | Llll Metrics \& More | 国 Article Recommendations | sl Supporting Information

ABSTRACT: The polarization response of a coplanar electrochemical capacitor covered with an ionic liquid as the electrolyte has been examined using a combination of two powerful analytic techniques, X-ray photoelectron spectroscopy (XPS) and scanning electron microcopy (SEM). Spatiotemporal distribution of the ionic liquid surface potential, upon DC or AC (square wave) biasing, has been monitored via chemical element binding energy shifts using XPS and secondary electron intensity variations using SEM. SEM's high spatial resolution and speedy imaging together with application of a data mining algorithm made mapping of the surface potential distribution across the capacitor possible. Interestingly, despite the differences in the detection principles, both techniques yield similar polarization relaxation time constants. The results demonstrate the power of a synergistic combination of the two techniques with complementary capabilities and pave the way to a deeper understanding of liquid/solid interfaces and for performance evaluation and diagnostics of electrochemical devices.

\section{INTRODUCTION}

Ionic transport controls the electrochemical processes and operation of the energy generation and storage devices ${ }^{1,2}$ and biological and environmental systems. ${ }^{3,4}$ Ionic liquids (ILs) have been introduced as a recent and welcomed addition to the conventional electrolytes for a number of promising applications. ${ }^{5}$ The basic understanding is that the physicochemical processes within the $1-100 \mathrm{~nm}$ thin layer at the solid/liquid interfaces play the most important role in controlling the performance of such devices. ${ }^{6-8}$ The structure and dynamics of the electrified interfaces have been debated for more than one and a half centuries and revisited recently in theoretical work by Bazant and Kornyshev. ${ }^{9-14}$ Over the last three decades, significant experimental and theoretical efforts have been devoted to understanding the composition and structure and the numerous other properties of these ultranarrow interfaces. In these efforts, in addition to the well-established electrochemical metrology, advanced optical, microscopy, and scattering techniques have been utilized. ${ }^{15-30}$ These have revealed that the screening dynamics evolving on multiple time and length scales are due to various diffusion and relaxation processes. This has been also corroborated by simulations. $^{31,32}$ These studies have revealed that the polarization of ILs includes medium-slow $(0.1 \mathrm{~s}-10 \mathrm{~s})$ and ultraslow $\left(10^{2}-10^{4} \mathrm{~s}\right)$ evolutions, in addition to the fast electrical double-layer (EDL) formation (1-10 ns), which also proceeds on short $(1-10 \mathrm{~nm})$, medium $(<1 \mu \mathrm{m})$, and long

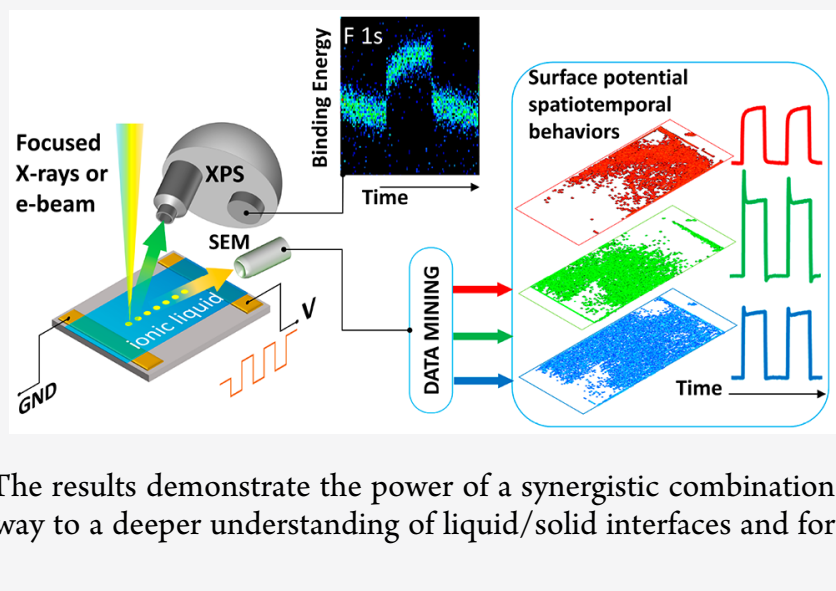

(>100 $\mu \mathrm{m}$ ) length scales. ${ }^{33-40}$ Therefore, for extended IL devices, the information reflecting not only the solid/liquid interface but also the bulk liquid and electrodes must be accounted for comprehensive analyses.

Operando analytic and imaging techniques have also been implemented to establish a deeper understanding of the interfacial properties. Among them, the techniques utilizing electron emission are particularly suitable for providing the information related to the electrical potential evolution during charge redistribution. In particular, two techniques with complementary capabilities, scanning electron microcopy (SEM) with its higher lateral resolution, and X-ray photoelectron spectroscopy (XPS) with its high chemical specificity, stand out. ${ }^{41}$

In both techniques, the measured signal depends on the local surface potential (Figure 1a). In SEM, the surface potential variations are observed as a reduction/enhancement of the secondary electron (SE) intensity, while in XPS, the potential-induced variations in the measured photoelectron kinetic energy manifest as binding energy (BE) shifts of all

Received: July 2, 2021

Accepted: September 8, 2021

Published: September 21, 2021 
a)

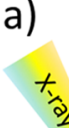

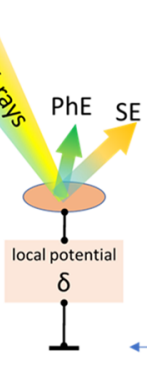
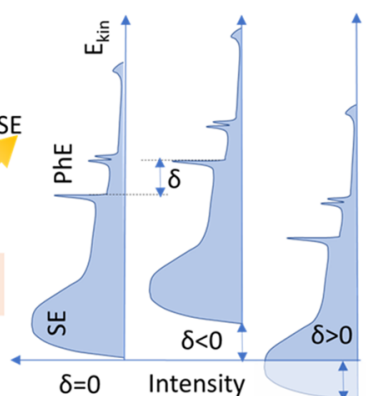

b)

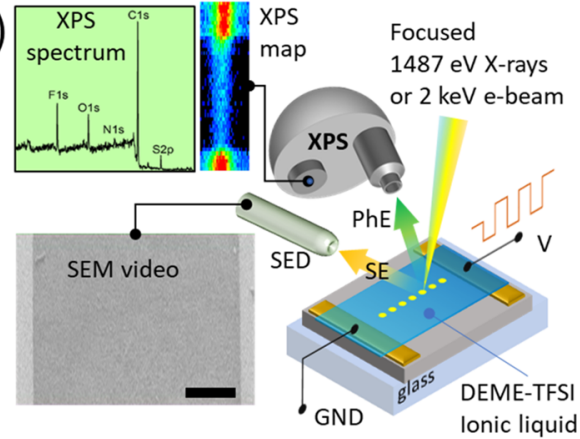

Figure 1. (a) Diagram explaining the sensitivity of the measured $\mathrm{SE}$ intensity and photoelectron $(\mathrm{PhE})$ kinetic energies $\left(E_{\text {kin }}\right)$ to the local surface potential $(\delta)$ with respect to the detector (grounded: GND). Low-energy SEs are accelerated toward the detector at negative $\delta$, but a significant fraction of SE cannot escape the sample under the positive $\delta$. Similarly, the photoelectrons gain or lose their kinetic energy depending on the sign and value of the local potential. (b) XPS and SEM (separate chambers) setups for polarization studies of the lateral electrochemical device. SED stands for the SE detector. The scale bar in the SEM video panel corresponds to $1 \mathrm{~mm}$. See text for details.
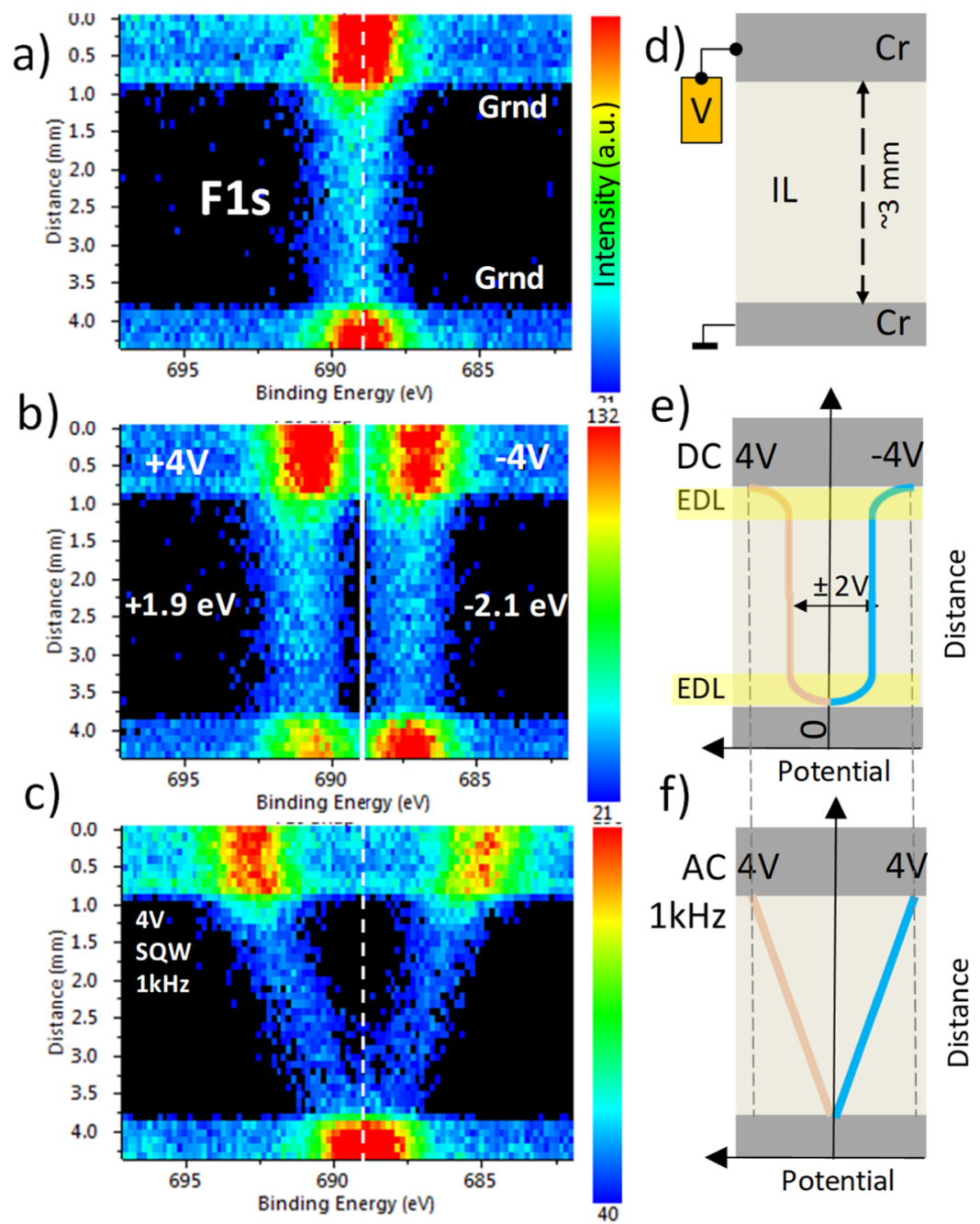

Figure 2. F 1s XP spectra recorded from the surface of the coplanar device along the center line when the top electrode is (a) grounded and (b) under $+4 \mathrm{~V}$ (left panel) and $-4 \mathrm{~V}$ DC bias (right panel) and (c) under AC $1 \mathrm{kHz}$ SQW excitations. (d-f) Corresponding steady-state potential distributions across the sample that cause the mentioned F 1s BE shifts.

peaks. The ability of SEM to probe the potential distribution across the surface is routinely used to study the doping profiles, ${ }^{42,43} \mathrm{p}-\mathrm{n}$ and Schottky barriers in semiconductors, ${ }^{44}$ for complementary metal-oxide-semiconductor device diagnostics, ${ }^{45}$ intercalation and formation of solid/electrolyte interface layers, ${ }^{46}$ electromigration in electrochemical devi- ces, $^{29}$ and more recently in liquid electrolytes. ${ }^{28}$ Electrical potential distribution/variations in solid/liquid electrolyte interfaces, as revealed by XPS, are even more informative since the sign, the magnitude, and also the variations in cation/ anion ratios are directly quantifiable. ${ }^{47-49}$ 


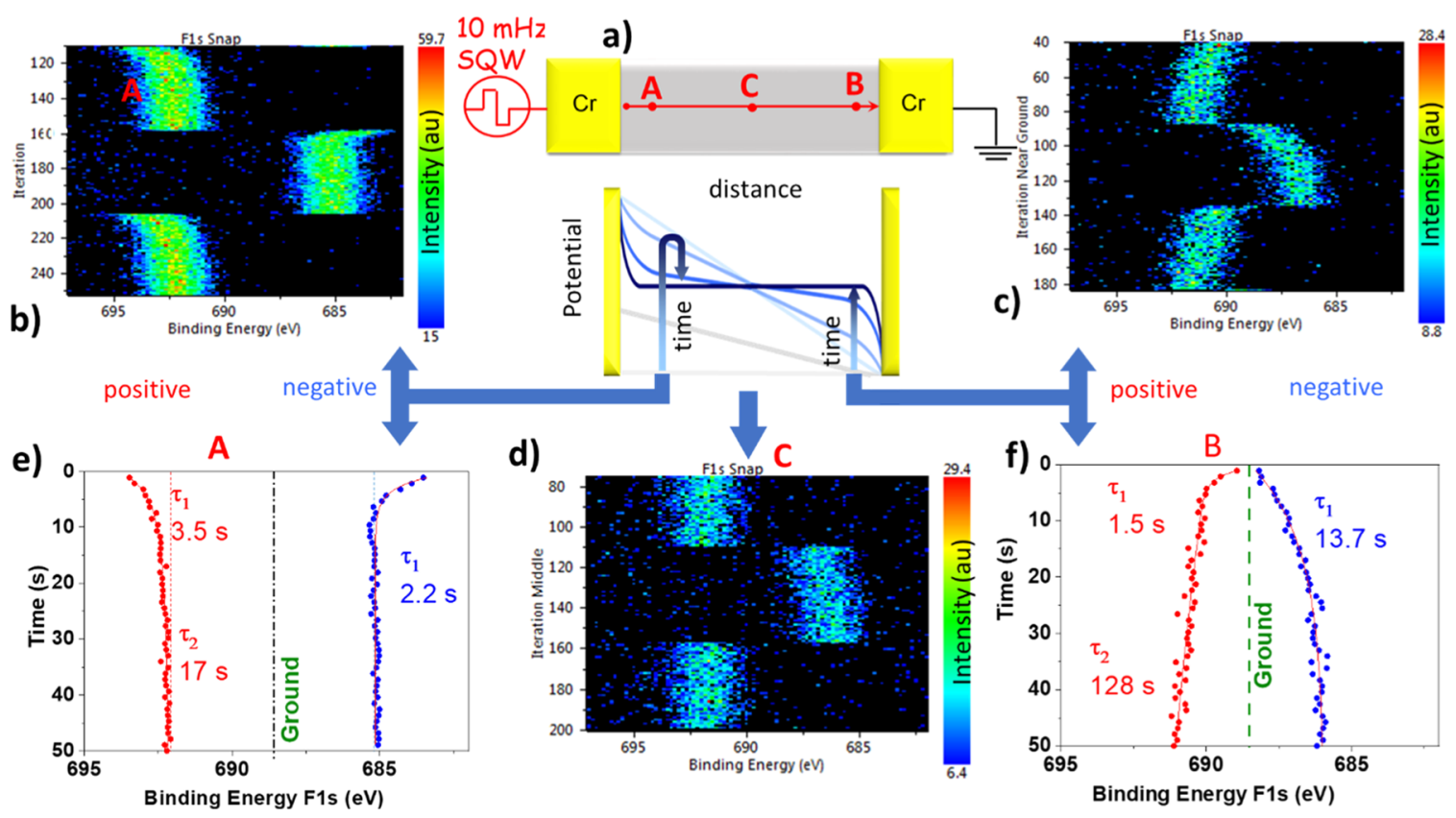

Figure 3. (a) Schematics of the device and potential evolution during step excitation of the left electrode. (b-d) F 1 s peak recorded under $10 \mathrm{mHz}$ $4 \mathrm{~V} \mathrm{SQW}$ excitation and using the snapshot mode with $1 \mathrm{~s}$ timesteps at three different lateral positions along the device. (e,f) Extracted BE evolutions at points $\mathrm{A}$ and $\mathrm{B}$, respectively.

Tsuda et al. have reported earlier on a combined SEM and XPS investigation of anodes of IL-based $\mathrm{Li}$ batteries, ${ }^{50}$ followed by a more recent report by Benayad et al. ${ }^{51}$ The potential distribution at the electrified liquid/solid interfaces has been recently probed via careful deconvolution of XPS signatures of the special molecular probes. ${ }^{52}$

This present work reports on a comparative XPS and SEM operando study of a model coplanar electrochemical device consisting of two metal electrodes deposited on a porous polyethylene membrane (PEM) film coated with an IL as the electrolyte medium. We have previously reported an XPS study of similar IL devices, demonstrating that the polarizationinduced electrical potential profiles can be followed on the macroscale, both as time-resolved (in the order of seconds) and also spatially resolved (on the millimeter scale) fashions, using both DC and AC actuations. ${ }^{53,54}$ A natural and desirable extension of this research is a combined study on the same material and device to address two questions pertaining to the similarities and the differences in the underlying detection principles and probe depths of the two techniques: (i) How comparable is the surface potential information obtained with these different techniques? (ii) What are the advantages of such a multimodal characterization?

\section{EXPERIMENTAL SECTION}

A two-electrode coplanar electrochemical capacitor device was fabricated by evaporating two parallel $\mathrm{Cr}$ strips onto a rectangular $20 \mu$ m-thick microfibrous PEM with a porosity of $43 \%$. Before the measurements, the membrane was impregnated with an IL DEME-TFSI, [N,N-diethyl- $N$ methyl-N-(2-methoxyethyl) a m moniu m-bis (trifluoromethanesulfonyl)imide], which bridged the two $\mathrm{Cr}$ electrodes (Figure 1b). Two separate vacuum chambers have been used for SEM and XPS measurements. For SEM studies, $2 \mathrm{keV}$ primary electrons and an Everhart-Thornley-type SE detector have been used. One electrode of the device was grounded, while a square wave $(\mathrm{SQW})$ bias $(3 \mathrm{~V}, 10 \mathrm{mHz})$ was applied to the second one during operando polarization SEM video recording with the frame rate of $1 \mathrm{~Hz}$. Low magnification $(\times 35)$ and low beam current $(100 \mathrm{pA})$ were selected to avoid beam-induced electrolyte damage and observe both electrodes within the same field of view. The grounded electrostatic shield (not shown here) with a slit exposing only $1 \mathrm{~mm} \times 4 \mathrm{~mm}$ of the sample area was placed above the sample surface to suppress the effect of the bias on the detected SE signal.

For spatially resolved XPS studies, monochromatic $\mathrm{Al} \mathrm{K} \alpha \mathrm{X}$ rays $(1486.68 \mathrm{eV})$ were used, which can be focused down to a $50 \mu \mathrm{m}$ spot. No X-ray- or electron beam-induced radiation damage could be observed during spectrum, image, or chemical map acquisition. The wiring of the sample was the same as in the SEM setup with DC or AC SQW biases ( $3 \mathrm{~V}, 10$ $\mathrm{mHz}$ and $4 \mathrm{~V}, 1 \mathrm{kHz}$ ) being applied to the nongrounded electrode.

\section{RESULTS AND DISCUSSION}

We first apply spatially resolved (spot size $50 \mu \mathrm{m}$ ) XPS to analyze the potential distribution along the central line of the device (Figure 1b). Although C 1s appears as the strongest feature within the XPS data (see the inset XPS spectrum panel in Figure $1 \mathrm{~b}$ ), it has a convoluted peak shape, incorporating contributions from both the membrane and the IL. Therefore, for tracking the electrical potential variations, we use $F$ 1s, representing the IL anion and having a relatively larger photoemission cross-section. In Figure 2, we summarize the typical lateral potential distribution derived from the operando XPS measurements during the DC or AC excitations. The F $1 \mathrm{~s}$ peak is at a $\mathrm{BE}$ of $688.5 \mathrm{eV}$ under grounded conditions but displays significant $\mathrm{BE}$ shifts under biasing, reflecting both spatial- and time-dependent local electrical potential developments. The application of a DC bias $U=+4 \mathrm{~V}$ or $U=-4 \mathrm{~V}$ induces spatially quasi-uniform but nonconforming (with respect to the applied bias) BE shifts along the device (Figure 
2b). Note that the steady-state BE shift value is ca. $U / 2$. Different BE shift patterns appear when larger than $100 \mathrm{~Hz}$ SQW excitation is applied to the top electrode. Here, a twosided (V-shaped) linear BE shift pattern can be observed, reflecting the positive and negative cycles of the square wave, recorded using the electron energy analyzer simultaneously. These variations are attributed to two extreme conditions: (i) the fully relaxed (DC, $t=\infty$ ) and (ii) the fast transient (AC) polarization of the IL between two coplanar electrodes. In the former case (DC), the BE shift reflects the potential distribution across the sample after all ionic relaxation processes in the coplanar IL capacitor are complete.

Under these conditions, approximately half of the potential drops at each of the EDLs formed symmetrically near the electrodes, thus leaving $\approx U / 2$ potential, uniformly distributed across the sample. Note that the width of the EDL is well below the spatial resolution of the XPS $(\approx 50 \mu \mathrm{m})$ and cannot be seen in the XPS maps.

On the other hand, the formation of the $\mathrm{V}$-shaped linear potential distribution in the case of $1 \mathrm{kHz} \mathrm{AC}$ excitations is remarkable and indicates that the screening of the electrode potential by the mobile ions (EDL formation at both electrodes) is not effective; hence, the ions in the IL can be seen as effectively frozen at these frequencies. ${ }^{53,54}$ Therefore, the corresponding polarization relaxation time of such an electrochemical device is well above the millisecond range.

To shed further light on the temporal evolutions of the polarization process, the $\mathrm{BE}$ position of the $\mathrm{F}$ 1s peak was fastsampled via application of $10 \mathrm{mHz}$ SQW excitations with $1 \mathrm{~s}$ intervals using the snapshot mode of data collection from a single point at three locations A, B, and C, along the device (Figure $3 a)$. The results are shown in Figure $3 b-d$, which reveal two important findings. First, the temporal response of the IL, in general, can be fitted with two time constants $\left(\tau_{1}\right.$ and $\left.\tau_{2}\right)$, one in the range of seconds and the other one being much longer, as depicted in Figure 3e,f. Second, a clear asymmetry is evident with respect to the polarity of the applied bias; negative biasing (lower $\mathrm{BE}$ ) results in a single short time relaxation constant, while the positive one (higher $\mathrm{BE}$ ) requires two time constants for fitting. Both findings are surprisingly well corroborated with the SEM data, as is discussed below.

In Figure 4, we summarize the SEM data collected under a $10 \mathrm{mHz}$ frequency $3 \mathrm{~V}$ SQW excitation by recording a video file of the SE yield (SEY) dependence on two spatial $(x, y)$ and time coordinates $(t)$. In a video frame shown in Figure 4a, the local SEY from the surface of the IL apparently depends on the bias applied to the left (right) electrode, where a higher (or lower) yield is observed for the areas with the higher negative (or positive) potential. The electrodes can also be seen through the thin layer of the IL because of the type II SEs. However, the shown video frame has low contrast and is almost featureless between the electrodes.

To better understand the observed polarization behavior, the SEM video data have been analyzed in the following way. First, the time-dependent dataset of the $\operatorname{SE}$ intensity, $\operatorname{SEY}(x, y, t)$, has been averaged along the dimension running parallel to the electrodes to yield a visualizable $2 \mathrm{D}$ map. The resultant map is shown in Figure $4 \mathrm{c}$ and manifests a combined spatiotemporal polarization behavior. The voltage waveform applied to the left electrode and consisting of alternating $\pm 3 \mathrm{~V}$ square pulses is shown in Figure $4 \mathrm{~d}$. The IL film becomes laterally polarized upon excitation. As it can be seen in Figure 4c, the most significant variations occur near the biased electrode, and
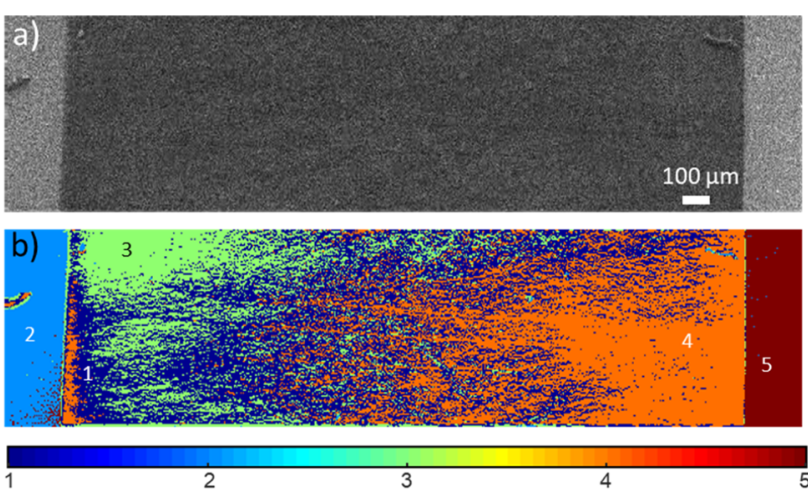

C) SEY Intensity $\times 10^{3}$ (a.u.)

d)
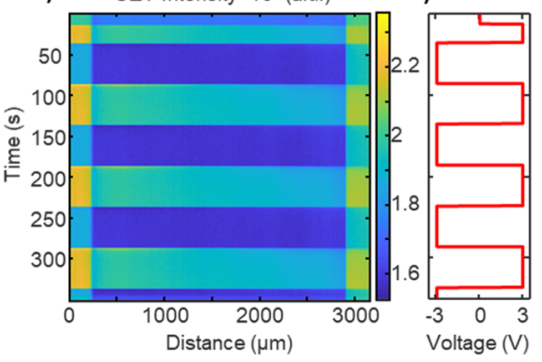

e)

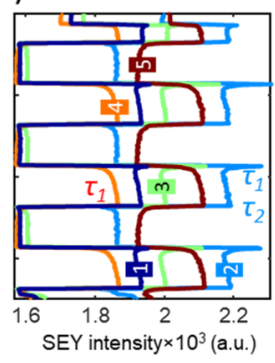

Figure 4. SEM imaging: (a) SEM video snapshot of the device with the biased electrode on the left and grounded electrode on the right; (b) $k$-means clustering loading map for five relaxation behaviors; (c) SEY intensity time-distance map obtained by averaging SEM video data along the spatial direction running parallel to the electrodes; (d) AC bias voltage waveform used in this experiment; (e) mean SEY responses for each of the $1-5$ clusters shown in (b); the same time axis in $(c-e)$.

response gradually vanishes toward the center of the device in proportion to the proximity to the biased electrode. Similar to XPS BE data, the temporal profile of SEY also exhibits a spatial dependence with the locations closer to the grounded electrode displaying a growing signal, and with those closer to the biased electrode, a more complex response is observed, with an initial rising spike followed by a slow decay. In the middle of the film, the SEY signal gradually changes from one type to another. Interestingly, both grounded and biased metal electrodes also manifest polarization behavior upon excitation. The latter is due to complete coverage of the electrodes with a thin layer of IL.

To extract the full content of the SEM video dataset in a visualizable form, we apply statistical and clustering algorithms for SEM video processing. Principal component analysis (PCA) in principle can do this, but its results are not easy to interpret meaningfully because the PCA algorithm is tuned to find statistically significant differences in the data while ignoring physical constraints. To make sure the results are physically meaningful, we employ the $k$-means clustering algorithm, ${ }^{55}$ which separates the $\operatorname{SEY}(x, y, t)$ spatiotemporal dataset into a user-defined number of clusters that have similar polarization temporal behaviors. The spatial distribution of the found clusters (behaviors) is displayed by mapping them by color. Figure $4 \mathrm{~b}$ shows a loading map for five clusters (see ref 56 on how to determine the optimal number of behavioral components). The corresponding color-coded mean $\operatorname{SEY}(t)$ polarization behaviors are shown in Figure 4e. Figure $4 \mathrm{~b}, \mathrm{e}$ presents all the information initially present in the original dataset in a form that is easy to percept and interpret. 
All the clusters' curves (Figure 4e) follow the applied square pulse voltage waveform, however, each with its own unique peculiarities. Cluster 5 (red) is present in the grounded electrode and shows a rising-shaped response with a relatively high average SEY value, due to the high SEY of the metal and its bias. Cluster 4 (orange), present in the film's region close to the grounded electrode, displays a similar behavior with a lower average SEY value. This cluster's spatial distribution reveals an existence of filamentary protrusions and a very narrow strip by the biased electrode. The remaining three clusters, localized in the biased electrode and in the film close to it, show strong polarization spikes at each bias onset. These clusters' responses differ in the magnitude of the spikes, with the largest present in clusters 2 (light blue) and 3 (green). These findings corroborate the SEM observations that the electrodes were covered with a thin layer of IL, and the yield of the escaping SEs was affected by the liquid EDL formed over the electrode, which responded to the applied bias. Interestingly, a defect (particle) is visible on the biased electrode, where components 1, 3, and 4 are present (Figure $4 \mathrm{~b}$ ), indicating that the particle is electrically decoupled from the electrode. In addition, the component distribution across the film is nonuniform, highlighting a fan-shaped structure invisible in the individual SEM images ( $c f$. Figure 4a,b). Note that the individual video frames have too low contrast to show this nonuniformity. These observations of the filamentary and other inhomogeneities in polarization behavior across the sample (Figure $4 \mathrm{~b}$ ) suggest that the captured polarization dynamics is most probably related to the structure of the IL impregnating the PE film. Defects and/or wetting particularities may result in the IL thickness irregularity across the film, therefore causing the observed effects.

While the raw SEM video data are too noisy to extract the local polarization relaxation time constants with acceptable precision, the $k$-means clustering and their time responses in Figure $4 \mathrm{e}$ provide such an opportunity. Moreover, $k$-means clustering allowed the mapping of the spatial distribution of these time constants and the corresponding behaviors (Figure $4 \mathrm{~b})$. As in the case of XPS results, the relaxation processes can be fitted with two [slow $\left(\tau_{1}\right)$ and very slow $\left.\left(\tau_{2}\right)\right]$ time constants in the order of seconds and few tens of seconds correspondingly (Figure 4e; see also Table $\mathrm{S} 1$ in the Supporting Information). Here, again, one can observe an interesting phenomenon that positive biasing requires 2 times constants to fit the data, presumably suggesting an asymmetry in diffusivities of cations and anions.

Although the observables in XPS (the BE shifts) and SEM (the SEY) of the same device are very different, their temporal and spatial variations are remarkably similar, both revealing the presence of slow $(1-10 \mathrm{~s})$ and ultraslow $(>10 \mathrm{~s})$ polarization relaxation time constants, although the origin and values of the observed time constants depend on the composition of the IL, device geometry, and detection mechanism and are beyond the scope of the current study. For preliminary explanations, we can invoke the consideration of Bazant et al. developed ${ }^{9}$ for the IL parallel-plate capacitor geometry. Namely, they introduced three characteristic time constants, (i) $\tau_{\mathrm{D}}=\left(\lambda_{\mathrm{D}}\right)^{2} / D$, (ii) $\tau_{\mathrm{C}}=$ $\left(\lambda_{\mathrm{D}} L\right) / D$, and (iii) $\tau_{\mathrm{L}}=(L)^{2} / D$, associated with electrode polarization, diffusion across the cell, and diffusion charge dynamics, respectively. ${ }^{9}$ Here, $\lambda_{\mathrm{D}}$ is the Debye length, $L$ is the half-distance between electrodes, and $D$ is the diffusion constant of the ions. Due to the nanometer-scale width of the EDLs in ILs, $\tau_{\mathrm{D}}$ ought to be far smaller than the sampling rates used in this study. Therefore, the slow time constant observed in our experiments could be associated with the ion diffusion across the cell $\left(\tau_{\mathrm{C}}\right)$. The typical experimentally observed Debye length for ILs is in the order of $10 \mathrm{~nm}$. $7,8,27,38$ Taking $L \sim 1.5 \mathrm{~mm}$ for our electrochemical capacitor and the diffusion coefficient for the IL as $\approx 10^{-11} \mathrm{~m}^{2} \mathrm{~s}^{-1,38}$ we can estimate $\tau_{\mathrm{C}} \approx 1.5 \mathrm{~s}$, in a reasonably close agreement with $\tau_{1}$ for both XPS and SEM results considering the coplanar (not parallel plate) geometry of the capacitor. The even slower time constant $\tau_{2}$ observed in our experiments is most probably related to the inhomogeneity of the diffusion paths for ions and has no relevance to $\tau_{\mathrm{L}}$, which scales as $10^{5} \mathrm{~s}$ for our geometry.

\section{CONCLUSIONS}

In summary, we used XPS and SEM techniques to comparatively study the surface potential evolutions in a coplanar IL capacitor during device polarization. We found that despite the different detection principles, these two techniques provide reasonably well-corroborating results on polarization relaxation time constants, which are related to slow (seconds) ionic migration along the device length. The high energy resolution of XPS allows for a precise quantitative analysis of the surface potential, while the high lateral resolution of SEM is useful for spatial potential mapping. Data mining algorithms applied to SEM polarization video data enabled the extraction of inhomogeneities in the spatiotemporal polarization behavior across the sample. The latter is presumably related to the sample's morphological inhomogeneities and can be responsible for multiple time constants observed in the experiment. Overall, the work demonstrates the usefulness of combining these two techniques to study complex electrochemical phenomena in operando. We believe that our findings would foster further experimental and theoretical studies on similar systems and motivate the realization of a combined instrument in future.

\section{ASSOCIATED CONTENT}

\section{Supporting Information}

The Supporting Information is available free of charge at https://pubs.acs.org/doi/10.1021/acs.analchem.1c02779.

Additional SEM images for the sample characterization and details of statistical analysis of the SEM video data (PDF)

\section{AUTHOR INFORMATION}

\section{Corresponding Authors}

Sefik Suzer - Department of Chemistry, Bilkent University, Ankara 06800, Turkey; 이이.org/0000-0002-58662600; Email: suzer@fen.bilkent.edu.tr

Andrei Kolmakov - Physical Measurement Laboratory, National Institute of Standards and Technology, Gaithersburg, Maryland 20899, United States; 이이.org/ 0000-0001-5299-4121; Email: andrei.kolmakov@nist.gov

\section{Author}

Evgheni Strelcov - Physical Measurement Laboratory, National Institute of Standards and Technology, Gaithersburg, Maryland 20899, United States; Maryland Nano Center, University of Maryland, College Park, Maryland 20742, United States

Complete contact information is available at: https://pubs.acs.org/10.1021/acs.analchem.1c02779 


\section{Notes}

The authors declare no competing financial interest.

Any mention of commercial products in this manuscript is for information only; it does not imply recommendation or endorsement by the NIST.

\section{ACKNOWLEDGMENTS}

S.S. thanks TUBITAK for the financial support provided through the project no. 118Z902. E.S. acknowledges support under the Cooperative Research Agreement between the University of Maryland and the National Institute of Standards and Technology Center for Nanoscale Science and Technology, award 70NANB14H209, through the University of Maryland. The authors thank Dr. A. Tselev (University of Aveiro), Dr. S. Pookpanratana (NIST), and Dr. A. Vladar (NIST) for constructive suggestions.

\section{REFERENCES}

(1) Tian, Y.; et al. Chem. Rev. 2021, 121, 1623-1669.

(2) Chen, K.; et al. Acc. Chem. Res. 2021, 54, 632-641.

(3) Liu, R.; Zhao, S.; Liu, J. ACS Appl. Electron. Mater. 2021, 3, 101118.

(4) Page, A.; Perry, D.; Unwin, P. R. Proc. R. Soc. A 2017, 473, 20160889.

(5) Welton, T. Biophys. Rev. 2018, 10, 691-706.

(6) Bard, A. J.; Faulkner, L. R. Electrochemical Methods: Fundamentals and Applications, 2nd ed.; Wiley-Interscience: New York, 2000; p 864.

(7) Gebbie, M. A.; et al. Proc. Natl. Acad. Sci. U.S.A. 2013, 110, 9674-9679.

(8) Gebbie, M. A.; et al. Chem. Commun. 2017, 53, 1214-1224.

(9) Bazant, M. Z.; Thornton, K.; Ajdari, A. Phys. Rev. E: Stat., Nonlinear, Soft Matter Phys. 2004, 70, 021506.

(10) Kilic, M. S.; Bazant, M. Z.; Ajdari, A. Phys. Rev. E: Stat., Nonlinear, Soft Matter Phys. 2007, 75, 021502.

(11) Kilic, M. S.; Bazant, M. Z.; Ajdari, A. Phys. Rev. E: Stat., Nonlinear, Soft Matter Phys. 2007, 75, 021503.

(12) Bazant, M. Z.; Storey, B. D.; Kornyshev, A. A. Phys. Rev. Lett. 2011, 106, 046102.

(13) Storey, B. D.; Bazant, M. Z. Phys. Rev. E: Stat., Nonlinear, Soft Matter Phys. 2012, 86, 056303.

(14) Fedorov, M. V.; Kornyshev, A. A. Chem. Rev. 2014, 114, 29783036.

(15) Lockett, V.; et al. J. Phys. Chem. C 2008, 112, 7486-7495.

(16) Lockett, V.; et al. Phys. Chem. Chem. Phys. 2010, 12, 1249912512.

(17) Drüschler, M.; Huber, B.; Roling, B. J. Phys. Chem. C 2011, 115, 6802-6808.

(18) Zheng, J.; et al. J. Phys. Chem. C 2011, 115, 7527-7537.

(19) Jitvisate, M.; Seddon, J. R. T. J. Phys. Chem. Lett. 2018, 9, 126131.

(20) Hayes, R.; et al. J. Phys. Chem. C 2011, 115, 6855-6863.

(21) Atkin, R.; et al. J. Mol. Liq. 2014, 192, 44-54.

(22) Collins, L.; et al. Nat. Commun. 2014, 5, 3871.

(23) Hayes, R.; Warr, G. G.; Atkin, R. Chem. Rev. 2015, 115, 63576426.

(24) Baylam, I.; et al. Femtosecond Pulse Generation with VoltageControlled Graphene Saturable Absorbers. In Optical Properties of Graphene; World Scientific, 2017; pp 389-433.

(25) Uysal, A.; et al. J. Phys.: Condens. Matter 2015, 27, 032101.

(26) Chu, M.; et al. J. Phys. Chem. C 2017, 121, 3841-3845.

(27) Lee, A. A.; et al. Faraday Discuss. 2017, 199, 239-259.

(28) Strelcov, E.; et al. Nano Lett. 2020, 20, 1336-1344.

(29) Guo, H.; et al. ACS Appl. Mater. Interfaces 2020, 12, 5665056657.

(30) Lu, Y.-H.; et al. Nano Lett. 2019, 19, 5388-5393.

(31) Bedrov, D.; et al. Chem. Rev. 2019, 119, 7940-7995.

(32) Wang, Y.-L.; et al. Chem. Rev. 2020, 120, 5798-5877.
(33) Anaredy, R. S.; Shaw, S. K. Langmuir 2016, 32, 5147-5154.

(34) Ma, K.; Jarosova, R.; Swain, G. M.; Blanchard, G. J. Langmuir 2016, 32, 9507-9512.

(35) Ma, K.; Jarošová, R.; Swain, G. M.; Blanchard, G. J. J. Phys. Chem. C 2018, 122, 7361-7367.

(36) Fillaud, L.; et al. Langmuir 2018, 34, 3686-3693.

(37) Anaredy, R. S.; Shaw, S. K. J. Phys. Chem. C 2019, 123, 89758982.

(38) Perez-Martinez, C. S.; Perkin, S. Soft Matter 2019, 15, 42554265.

(39) Wang, Y.; Jarošová, R.; Swain, G. M.; Blanchard, G. J. Langmuir 2020, 36, 3038-3045.

(40) Richter, Ł.; et al. Phys. Rev. Lett. 2020, 125, 056001.

(41) Kolmakov, A.; et al. ACS Nano 2008, 2, 1993-2000.

(42) Sealy, C. P.; Castell, M. R.; Wilshaw, P. R. Microscopy 2000, 49, 311-321.

(43) Pfeiffer, H. C.; et al. J. Vac. Sci. Technol. 1981, 19, 1014-1018.

(44) Lu, Y.-J.; et al. ACS Nano 2013, 7, 7640-7647.

(45) Rosenkranz, R. J. Mater. Sci.: Mater. Electron. 2011, 22, 15231535.

(46) Strelcov, E.; et al. Nanoscale 2015, 7, 3022-3027.

(47) Camci, M. T.; et al. J. Phys. Chem. C 2018, 122, 11883-11889.

(48) Wang, P.; et al. EcoMat 2020, 2, No. e12023.

(49) Nemšák, S.; et al. J. Am. Chem. Soc. 2017, 139, 18138-18141.

(50) Tsuda, T.; et al. Electrochim. Acta 2018, 279, 136-142.

(51) Benayad, A.; et al. J. Phys. Chem. A 2021, 125, 1069-1081.

(52) Favaro, M.; et al. Nat. Commun. 2016, 7, 12695.

(53) Camci, M. T.; et al. Phys. Chem. Chem. Phys. 2016, 18, 2843428440.

(54) Gokturk, P. A.; Suzer, S. J. Phys. Chem. C 2021, 125, 94539460.

(55) Haykin, S. Neural Networks: A Comprehensive Foundation; Prentice Hall, 1999.

(56) Strelcov, E.; et al. ACS Nano 2014, 8, 6449-6457. 